\title{
Accuracy Increase of the Regional Labour Productivity Forecasting
}

\author{
Tatyana A. Burtseva* \\ Kaluga State University of K.E. Tsiolkovsky (Kaluga), Russian Federation MEPhl (Obninsk branch), Russian Federation \\ *Corresponding Email: tbur69@mail.ru
}

\author{
Anton A. Surkov \\ MEPhl (Obninsk branch), Russian Federation \\ Tatyana E. Timashkova \\ MEPhl (Obninsk branch), Russian Federation
}

\section{Doi:10.5901/mjss.2015.v6n6s7p63}

\begin{abstract}
The study applies the new techniques in forecasting regional labour productivity based on a combination of measuring instruments of individual factors. The weight coefficients selection method used provides accuracy increase of regional labour productivity forecasting. Methodological basis of research is a combination of number of statistical methods to forecast different economic indicators, such as the trend analysis, a method of exponential smoothing, and a method of multidimensional average. Research results suggest that the economic indicators of gross regional product and number of economically active population is suitable in characterizing the economy of an investment attractive region, such as Kaluga region. The study provides the practice oriented approach towards up-to-date identification of labour productivity change at the regional context.
\end{abstract}

Keywords: forecasting, labour productivity, regional productivity, Kaluga region.

\section{Introduction}

When forecasting economic processes the researcher solves a choice problem of the best forecast in order to apply its results afterward. The statistical methods applied to forecast the economic indicators often give significantly various results in spite of a high quality of the models made on their basis. In practice, the version of the forecast which usually is most acceptable is left, and all others are rejected that reduces the efficiency of forecasting results application since various forecasts use different information though characterize the same process, but in different ways. In this regard, there is a question of information mutual addition and, as a result, obtaining the general forecast, which would use all the available information concerning an economic process. The mutual addition of information formed the basis of modern methodology how to combine individual forecasts and obtain the combined forecast at the end (Wallis, 2010). Practical research of different outstanding scientists show that combination of forecasts surpasses individual forecasts in the accuracy (Armstrong, 2001; Clemen, 1986; Frenkel et al., 2015; Goodwin, 2009; Newbold \& Granger, 1974). At first sight there is a contradiction because of the fact that association with less effective forecast can reduce a mean square mistake (to increase forecast accuracy) in comparison with separate forecasts, but the association on the base of an optimum weight coefficients set allows to make it (Granger \& Ramanathan, 1984). This research is about a practical realization of economic indicators forecasts combination on the basis of the defined optimum set of the weight coefficients characterizing the economics of investment attractive region (Russia, Kaluga region). In addition, the research deals with the accuracy increasing of regional labour productivity forecasting; the importance of a solution to a productivity decrease problem in the short term is presented.

Forecasting accuracy increasing of the economic indicators in the regional economics particularly used for measurement of regional labour productivity. From a practical point of view, improvement of methodology to forecast regional labour productivity helps to increase a quality of regional planning and forecasting docs' preparation in order to make appropriate administrative decisions concerning the regional economic sphere. 


\section{Methodological and Informational Base of Research}

\subsection{Information base of research}

This research reveals a methodology application of combined forecast based on the indicators characterizing economics of the Kaluga region: the gross regional product (GRP) and a number of economically active population in order to receive the predicted level of regional labour productivity. Initial levels of indicators are taken from the site of Federal State Statistics Service (Rosstat) for the period 1996 - 2014. Levels of indicators, according to Rosstat, are in a public access and show an annual values selection of the indicator for the observed period: one million rubles - for GRP and one thousand people - for the number of economically active population in regional economics. Forecasting is made for 2015. To check the accuracy of forecasting the predicted values of the chosen indicators (as of 2015) are used. These values are reported in the Kaluga government resolution (the August 22nd, 2014 N 493 'On the social and economic development forecast for the Kaluga region as of 2015 and planning 2016 and 2017').

\subsection{Methods of forecasting and a way of the forecast combination}

For individual forecasts of indicators, common statistical methods were used, such as a method of exponential smoothing and a trend model method. The $5 \%$ accuracy level is obtained in both cases. The combination of forecasts is made on the basis of the weighted average from two individual forecasts. The weight coefficients increasing forecasting accuracy are offered. Selection of weight coefficients was carried out by the chosen criterion of forecasting accuracy on the basis of weight coefficients couples search.

The forecasts combination method on the basis of multidimensional average means the averaging of all used individual forecasts. Such combined forecast takes the form:

$F=\sum_{i=1}^{n} w f_{i}$

where $\mathrm{F}$ - the integrated forecast, $f_{i}$ - the individual forecast, and $w_{i}-$ a weight coefficient for the individual forecast to be included in the integrated forecast. Forecasting accuracy is estimated on the basis of an average percentage deviation of an indicator calculated values from the actual values during the defined time period (an approximation error).

\section{Results and Conclusions}

Results of individual forecasts and their combination in 2014 and in 2015 are presented in tables 1 and 3. Official data of the Kaluga Government forecast are also provided in tables, the forecast was made in the 2014. Thus, combination of forecasts showed the best results concerning the mean absolute error (MAE) in comparison with individual forecasts.

Table 1. Forecast of GRP of the Kaluga region, million rubles

\begin{tabular}{lccc}
\hline Method of forecasting & $\mathbf{2 0 1 4}$ & $\mathbf{2 0 1 5}$ & MAE \\
Trend model & 387257.2 & 449145 & -0.8 \\
$y=15225,14+54,25 t^{3}$ & & & \\
Exponential smoothing & 394103.5 & 461567.1 & 0.1 \\
$\mathrm{a}=0,8$ and $y=0,1$ & 394103.5 & 455356.1 & 0.3 \\
Combination of forecasts & 387589.0 & 453337.7 & - \\
\hline The Kaluga Government forecast & &
\end{tabular}

Exponential smoothing was carried out when using an exponential trend with smoothing parameters. The choice of parameters $\alpha=0.8$ and $y=0.1$ is provided by the smallest size of a mean-square error. Besides, both trend model, and exponential smoothing do not show any autocorrelation of the residual so we can speak about a reliability of models.

Selection of weight coefficients while making the combined forecast was limited by two factors - the weights have to be positive and their sum should not exceed 'one'. Search of possible combinations of weight coefficients for individual forecasts with a step 0.1 showed that if the individual forecast received on the trend model basis has a weight coefficient 0.6 and the individual forecast received on the exponential smoothing basis - 0.4, MAE for the combined forecast will be $0.02 \%$ (table 2).

The achieved distribution of weights does not surprise - the trend model showed better accuracy than exponential smoothing. Besides, it should be noted, that any distribution of weight coefficients for individual forecasts shows increase 
in accuracy of the integrated forecast concerning individual forecasts. The integrated forecast showed that value of Kaluga region GRP (as of 2015) would make 451423.2 million rubles, thus, MAE of the integrated forecast was at the level of $0.02 \%$ - below than that of individual forecasts. According to the Kaluga region Government, the forecast of GRP makes 453337.7 million rubles. As for the number of economically active population in Kaluga region, the best possible forecast was made via the trend model 'polynom of the sixth degree' (table 3).

Table 2. Accuracy of the combined forecast with various combinations of weight coefficients

\begin{tabular}{lcccc}
\hline Weight value & $0.1-0.9$ & $0.2-0.8$ & $0.3-0.7$ & $0.4-0.6$ \\
Mean absolute error, \% & 0.4 & 0.2 & 0.3 & 0.02 \\
Weight value & $0.6-0.4$ & $0.7-0.3$ & $0.8-0.2$ & $0.9-0.1$ \\
Mean absolute error, \% & 0.3 & 0.3 & 0.3 & 0.3 \\
\hline
\end{tabular}

Results of forecasting are placed in table 3. Selection of optimum weight coefficients showed that it is necessary to use the weight coefficient 0.7 for the individual forecast which is carried out on the basis of trend model and $0.3-$ for the individual forecast which is carried out on the basis of exponential smoothing: only this coefficients combination will provide MAE for the combined forecast at the lowest possible rate $-0.005 \%$.

Table 3. The economically active population number forecast: Kaluga region, one thousand persons.

\begin{tabular}{lccc}
\hline & Forecast as of 2015 & MAE & Government Forecast \\
\hline Trend model & & & \\
$y=607,9-96,62 t+39,2 t^{2}-7,04 t^{3}+$ & 537.6 & $-0.06 \%$ & \\
$0,62 t^{4}-0,026 t^{5}+0,0004 t^{6}$ & & & 552 \\
Exponential smoothing & 552.6 & $0.1 \%$ & - \\
Combination of forecasts & 542.1 & $0.005 \%$ & \\
\hline
\end{tabular}

On the basis of the received results predictive labour productivity level in the Kaluga region as of 2015 is estimated: a labour productivity level in the 2015 comparing with the last year will make 92\%, in other words, decrease in labour productivity level comparing with the last year is observed.

\section{Conclusion}

The results shown in the research prove that the forecasts combination on the basis of multidimensional average increases the accuracy of economic indicators forecasting. This conclusion does not differ from the opinion of authoritative scientists who say that thanks to a correct selection of weight coefficients and the optimum number of the integrated forecasts, the combined forecast reveals impressive results of accuracy (Bates \& Granger, 1969). Often, using simple methods of forecasting and combining them it is possible to achieve the same results as if applying complex methods, and sometimes - even better results.

\section{Acknowledgment}

The research is carried out with financial support of the Russian humanitarian scientific fund and Government of the Kaluga region, project No.15-12-40004a (p) "Factors and reserves of regional labor productivity growth identification".

\section{References}

Armstrong, J. S. (2001). Combining forecasts. Kluwer Academic Publishers.

Bates, J.M., \& Granger, C.W.J. (1969). The combination of forecasts. Operational Research Quarterly, 20, 319-325.

Clemen, R. T. (1986). Linear constraints and the efficiency of combined forecasts. Journal of Forecasting, 5, 31-38.

Goodwin, P. (2009). New evidence on the value of combining forecasts. Foresight, 12, 33-35.

Granger, C.W.J., \& Ramanathan, R. (1984). Improved methods of combining forecasts. Journal of Forecasting, 3, $197-204$.

Newbold, P., \& Granger C.W.J. (1974). Experience with forecasting univariate time series and the combination of forecasts. Journal of the Royal Statistical Society, 137, 131-164.

Wallis, K.F. (2010). Combining Forecasts - Forty Years Later. 6th Eurostat Colloquium on Modern Tools for Business Cycle Analysis, 
2010, 1-17.

Frenkel, A., Rayskay, N., Burtseva, T., \& Surkov, A. (2015). Improving accuracy of forecasting investment by combining different forecasts. Bulletin IEP them. Gaidar, 01(1)5, 48-50. 